\title{
Расчеты из первых принципов электронного спектра и плотности состояний кристаллов $\mathrm{TIFeS}_{2}$ и $\mathrm{TIFeSe}_{2}$
}

\author{
(C) Н.А. Исмайылова ${ }^{1}$, Г.С. Оруджев ${ }^{1,2}$, С.Г. Джабаров ${ }^{1,2}$ \\ ${ }^{1}$ Институт физики Национальной академии наук Азербайджана, \\ Az-1143 Баку, Азербайджан \\ ${ }^{2}$ Азербайджанский технический университет, \\ Az-1073 Баку, Азербайджан \\ ฯ E-mail: ismayilova_narmin_84@mail.ru
}

(Получена 20 сентября 2016 г. Принята к печати 30 сентября 2016 г.)

\begin{abstract}
Представлены результаты расчетов из первых принципов в рамках теории функционала плотности электронного спектра кристаллов $\mathrm{TlFeS}_{2}$ и $\mathrm{TlFeSe}_{2}$ в антиферромагнитной фазе. Исследованы происхождения зон из $s$-, $p$-, $d$-электронных состояний атомов $\mathrm{Tl}, \mathrm{Fe}, \mathrm{S}, \mathrm{Se}$ Установлено, что в этой фазе кристаллы обладают полупроводниковыми свойствами. Величины запрещенной зоны 0.05 эB $\left(\mathrm{TlFeS}_{2}\right)$ и 0.34 эB $\left(\mathrm{TlFeSe}_{2}\right)$ соответственно.
\end{abstract}

DOI: $10.21883 / F T P .2017 .04 .44341 .8290$

\section{1. Введение}

В последнее время возрос интерес к исследованиям цепочечных полупроводников. Интерес связан с широким спектром физических явлений, наблюдаемых в этих соединениях: магнитные фазовые переходы, антиферромагнитные и парамагнитные состояния, химическое и зарядовое упорядочение ионов и сильной анизотропности физических свойств вдоль различных кристаллографических направлений. Одним из представителей таких кристаллических веществ являются тройные соединения составов $\mathrm{TlFeS}_{2}$, входящие в общую группу полупроводников типа $\mathrm{TlMeX}_{2}$ (где $\mathrm{Me}-3 \mathrm{~d}$-металл, $X=\mathrm{S}, \mathrm{Se}, \mathrm{Te}$ ), обладающих магнитными свойствами [1-4].

Соединения $\mathrm{TlFeS}_{2}$ и $\mathrm{TlFeSe}_{2}$ обладают моноклинной структурой с пространственной симметрией $C 2 / m$. Значения параметров элементарной ячейки в нормальных условиях для $\mathrm{TlFeS}_{2}$ составляют: $a=11.646(1) \AA, \quad b=5.308(2) \AA, \quad c=6.831(3) \AA$, $\beta=116.7(4)^{\circ}$; для $\mathrm{TlFeSe}_{2}$ составляют: $a=11.998(1) \AA$, $b=5.498(9) \AA, c=7.108(8) \AA, \beta=118.2(3)^{\circ}$ [5-7]. Тетраэдры $\mathrm{FeX}_{4}$ расположены вдоль моноклинной кристаллографической оси $b$. В центре тетраэдров находятся ионы железа $\mathrm{Fe}^{3+}$, а в вершинах расположены ионы халькогена $\mathrm{S}(\mathrm{Se})^{2-}$. Кроме прямого обмена $\mathrm{Fe}-\mathrm{Fe}$, магнитное взаимодействие между ионами железа вдоль цепи тетраэдров осуществляется по цепочке атомов $\mathrm{Fe}-\mathrm{S}(\mathrm{Se})-\mathrm{Fe}$, образующих определенный угол [4].

Эксперименты по порошковой нейтронной дифракции показывают, что при температуре ниже $210 \mathrm{~K}$ в $\mathrm{TlFeS}_{2}$, $295 \mathrm{~K}$ в $\mathrm{TlFeSe} \mathrm{F}_{2}$ наблюдается антиферромагнитная фаза. При фазовом переходе резко изменяется угол моноклинности $\beta$, при увеличении температуры - уменьшается. $\mathrm{C}$ понижением температуры от комнатной до температуры фазового перехода межатомное расстояние $\mathrm{Fe}-\mathrm{Fe}$ быстро уменьшается и достигает в точке фазового перехода величины $l_{\mathrm{Fe}-\mathrm{Fe}} \sim 2.501(3) \AA \AA$. При дальнейшем понижении температуры межатомное расстояние $\mathrm{Fe}-\mathrm{Fe}$ уменьшается слабо [5].
В работе [5] установлено, что магнитные моменты ионов $\mathrm{Fe}$ в низкотемпературной антиферромагнитной фазе в плоскости $(a b)$ ориентированы антипараллельно вдоль оси $b$. При высоких давлениях $\mathrm{TlFeS}_{2}$ и $\mathrm{TlFeSe}_{2}$ сохраняют моноклинную кристаллическую структуру, магнитная фаза не наблюдается [7].

В настоящей работе проведено исследование кристаллической структуры и спектра плотности состояний электронов соединений $\mathrm{TlFeS}_{2}$ и $\mathrm{TlFeSe}_{2}$ методом функциональной плотности.

\section{2. Метод расчета и кристаллическая структура}

В статье представлены результаты расчетов из первых принципов в рамках теории функционала плотности [8] электронного спектра кристаллов $\mathrm{TlFeS}_{2}, \mathrm{TlFeSe}_{2}$ в антиферромагнитной фазе. Электронная структура кри-

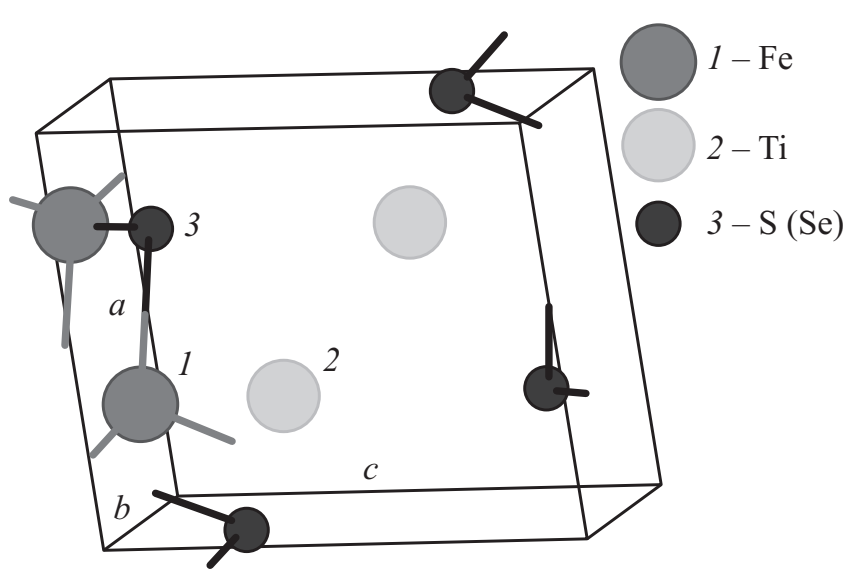

Рис. 1. Кристаллическая структура примитивной ячейки одномерного соединения $\mathrm{TlFeS}_{2}\left(\mathrm{TlFeSe}_{2}\right)$. Сферы с малого, среднего и большого размера представляют собой атомы $\mathrm{S}(\mathrm{Se}), \mathrm{Fe}$, и $\mathrm{Tl}$ соответственно, а $a, b, c$ параметры элементарной ячейки. 

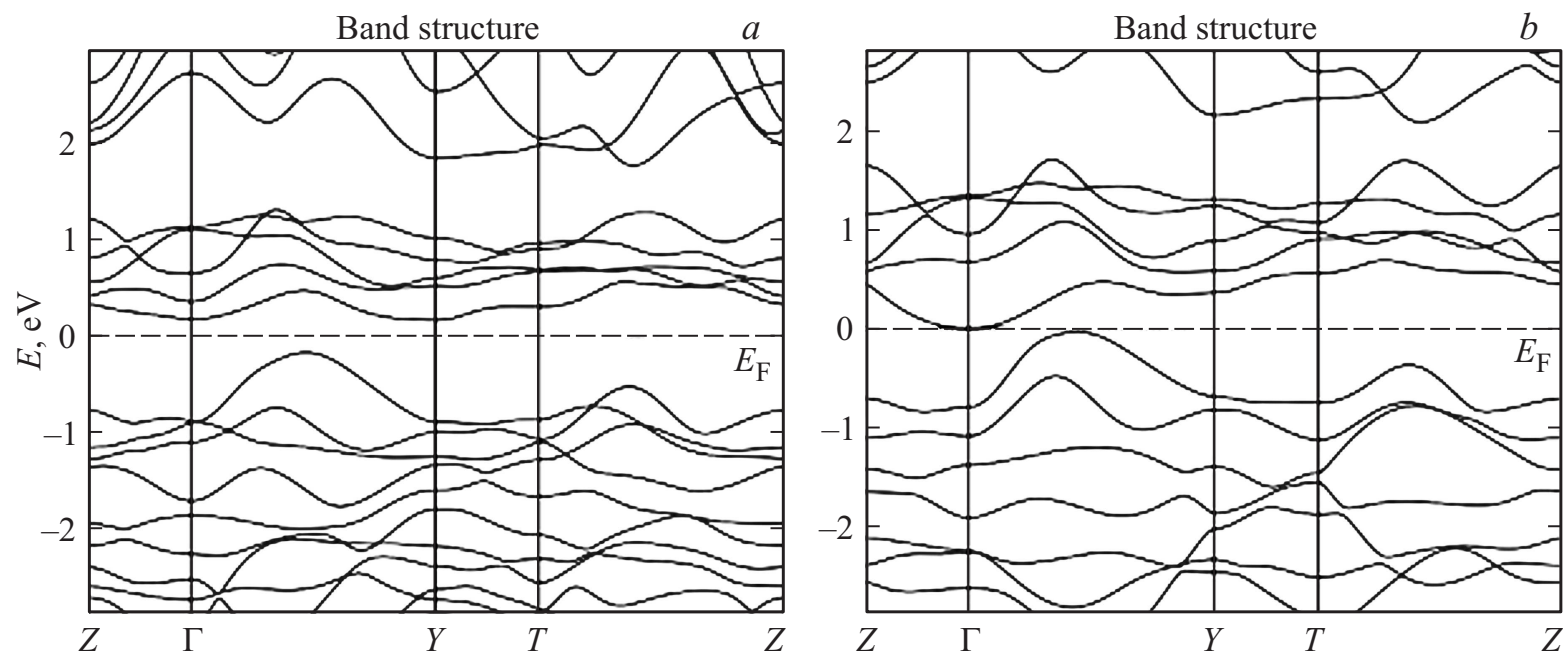

Рис. 2. Рассчитанная зонная структура с приближением $\mathrm{SGGA}$ для $\mathrm{TlFeSe}_{2}(a)$ и $\mathrm{TlFeS}_{2}(b)$ в антиферромагнитной фазе вблизи уровня Ферми.

сталлов изучена с использованием пакета программ Quantum Wise - Atomistix Tool Kit [9] в приближении SGGA (спин-обобщенная градиентная аппроксимация) [10] обменно-корреляционным потенциалом, электрон-ионные взаимодействия учтены через псевдопотенциал FHI (Fritz Haber Institute) [11]. Расчеты показывают, что элементарные ячейки (рис. 1) полупроводниковых кристаллов соединений $\mathrm{TlFeS}_{2}, \mathrm{TlFeSe}_{2}$ со слоисто-цепочечной структурой состоят из слоевых пакетов $\mathrm{Fe}_{4} \mathrm{~S}_{10}, \mathrm{Fe}_{4} \mathrm{Se}_{10}$, которые в свою очередь образованы тетраэдрами $\mathrm{FeS}_{4}, \mathrm{FeSe}_{4}$. В центре тетраэдров находятся ионы железа $\mathrm{Fe}^{3+}$, а в вершинах расположены ионы халькогенов $\mathrm{S}^{2-}, \mathrm{Se}^{2-}$. Пирамиды образованных слоев обращены друг к другу вершинами, и в получившихся таким образом тригональных полостях располагаются атомы Tl. Это полностью согласуется с предыдущими экспериментальными результатами [4]. Элементарная ячейка кристалла содержит четыре формульные единицы. Преобразование одного атома Fe в другой происходит операцией инверсии. Атомы Tl, S и Se расположены в стандартных положениях 4(i), а атомы $\mathrm{Fe}$ в положении $4(g)$. Количество электронов, рассматриваемых как валентные электроны, составляло 3 для $\mathrm{Tl} 6 s^{2} 6 p^{1}, 8$ для $\mathrm{Fe} 3 d^{6} 4 s^{2}, 6$ для $\mathrm{S}, \mathrm{Se} 3 s^{2} 3 p^{4}$. Для определения координат атомов и параметров решетки примитивной ячейки $\mathrm{TIFeS}_{2}, \mathrm{TIFeSe}_{2}$ межатомные силы были оптимизированы до 0.001 эВ/Ä, тензор механических напряжений был оптимизирован до 0.001 эВ/ $\AA^{3}$. Оптимизированные значения параметра элементарной ячейки составили $a=11.846 \AA, b=5.04 \AA, c=6.768 \AA$, $\beta=117.343^{\circ}$ для $\mathrm{TIFeS}_{2}, a=12.176 \AA, b=5.431 \AA$, $c=7.262 \AA, \beta=118.364^{\circ}$ для $\mathrm{TIFeSe}_{2}$ соответственно, что хорошо согласуется с результатами работы [5-7].

\section{3. Обсуждение результатов}

Анализируя результаты экспериментов по магнитной восприимчивости и зависимости электрического

Рассчитанные в SGGA приближении с FHI псевдопотенциалом локальные магнитные моменты атомов ячейки $\mathrm{TIFeS}_{2}$, TIFeSe 2 и суммарный магнитный момент в АФМ и ФМ фазах при разных ориентациях спина

\begin{tabular}{|c|c|c|c|c|c|c|c|c|}
\hline \multirow{4}{*}{$\begin{array}{c}\text { Соединение } \\
\text { Фаза } \\
\begin{array}{c}\text { Направление } \\
\text { спина }\end{array}\end{array}$} & \multicolumn{4}{|c|}{$\mathrm{TlFeS}_{2}$} & \multicolumn{4}{|c|}{$\mathrm{TlFeSe}_{2}$} \\
\hline & \multicolumn{2}{|c|}{$\mathrm{A} \Phi \mathrm{M}$} & \multicolumn{2}{|c|}{$\Phi \mathrm{M}$} & \multicolumn{2}{|c|}{ АФМ } & \multicolumn{2}{|c|}{$\Phi \mathrm{M}$} \\
\hline & Спин & Спин & Спин & Спин & Спин & Спин & Спин & Спин \\
\hline & вверх & вниз & вверх & вниз & вверх & вниз & вверх & вниз \\
\hline $1 \mathrm{TI}$ & 1.605 & 1.605 & 1.632 & 1.613 & 1.666 & 1.666 & 1.721 & 1.635 \\
\hline $2 \mathrm{TI}$ & 1.605 & 1.605 & 1.632 & 1.613 & 1.666 & 1.666 & 1.721 & 1.635 \\
\hline $3 \mathrm{Fe}$ & 5.202 & 2.848 & 4.341 & 3.733 & 2.357 & 5.503 & 5.341 & 2.52 \\
\hline $4 \mathrm{Fe}$ & 2.848 & 5.202 & 4.341 & 3.734 & 5.502 & 2.358 & 5.341 & 2.52 \\
\hline $5 \mathrm{~S}$ & 2.942 & 2.942 & 2.959 & 2.892 & 2.956 & 2.955 & 2.958 & 2.936 \\
\hline $6 \mathrm{~S}$ & 2.942 & 2.942 & 2.959 & 2.892 & 2.948 & 2.948 & 2.959 & 2.936 \\
\hline $7 \mathrm{~S}$ & 2.928 & 2.928 & 2.942 & 2.888 & 2.948 & 2.948 & 2.979 & 2.909 \\
\hline $8 \mathrm{~S}$ & 2.928 & 2.928 & 2.942 & 2.888 & 2.948 & 2.948 & 2.979 & 2.909 \\
\hline$\mu_{\text {total }}(\mu$ Bohr $)$ & \multicolumn{2}{|l|}{2.020} & \multicolumn{2}{|c|}{1.495} & \multicolumn{2}{|c|}{0} & \multicolumn{2}{|c|}{5.999} \\
\hline
\end{tabular}



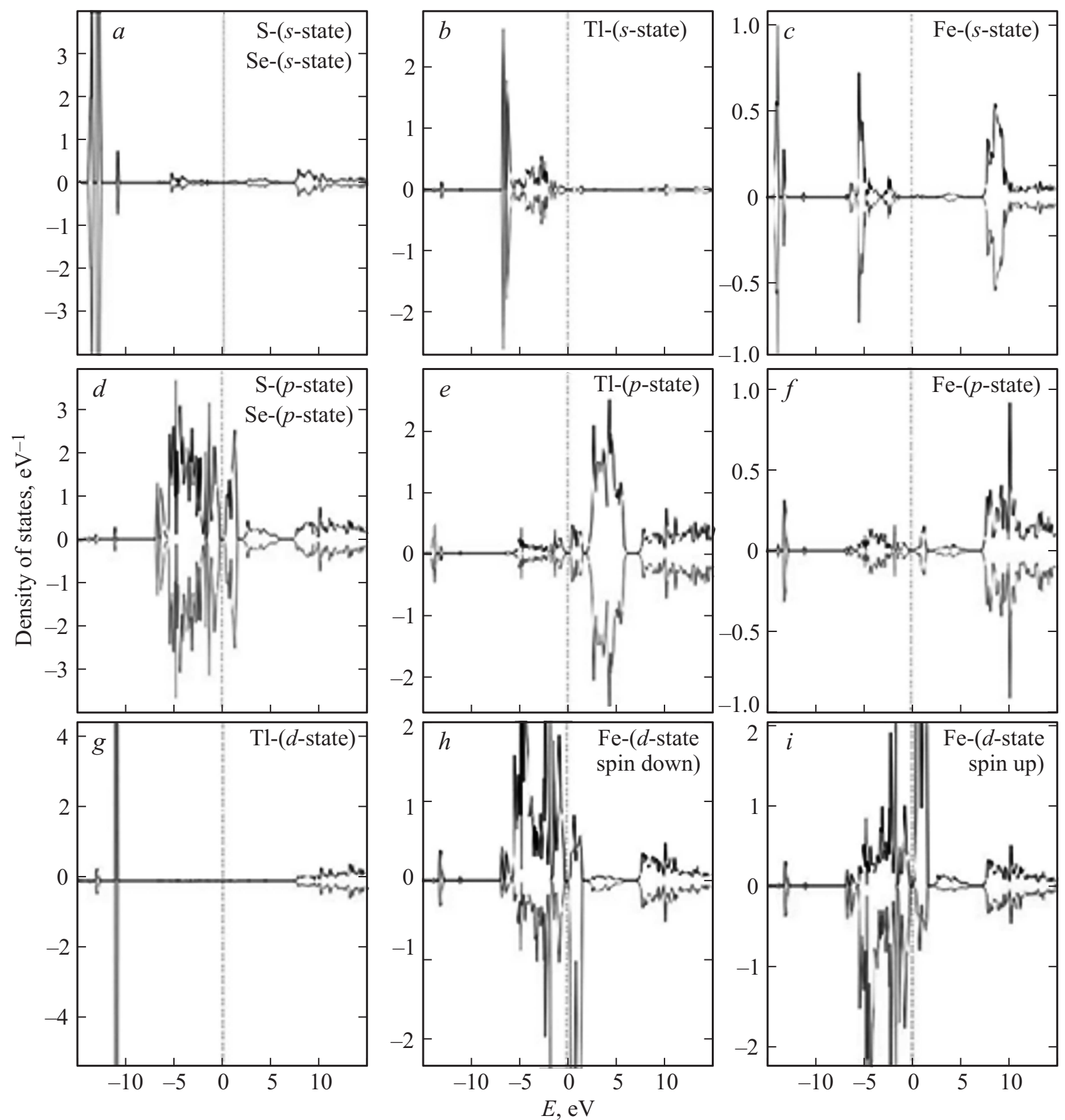

Рис. 3. Локальные плотности состояний $\mathrm{TlFeS}_{2}, \mathrm{TlFeSe}_{2}$ для $\mathrm{S}(\mathrm{Se}): s-(a), p-(b)$, для $\mathrm{Tl}: s-(c), p-(d), d-(e)$, для $\mathrm{Fe}$ $s-(f), p-(g)$ и плотность состояний „spin uр“ и „spin down“ поляризаций для Fe: $d-(h), d-(i)$.

сопротивления от температуры монокристаллов $\mathrm{TlFeS}_{2}$, $\mathrm{TIFeSe}_{2}[4]$, было определено, что эти кристаллы являются антиферромагнитными полупроводниками. Проведенные расчеты методом теории функционала плотности в приближении SGGA действительно подтверждают, что эти кристаллы являются антиферромагнитными полупроводниками. Так как полная энергия на кристаллической ячейки в антиферромагнитной фазе в кристале $\mathrm{TlFeS}_{2}$ : -4115.50249 эВ (TIFeSe $2:-4255.7188$ эВ) ниже, чем полная энергия ячейки в ферромагнитной фазе -4115.09247 эВ ( $\mathrm{TIFeSe}_{2}:-4254.9318$ эВ), то в этой фазе кристаллы являются полупроводниковыми соединени- ями. На рис. 2 показаны рассчитанные зонные структуры кристаллов $\mathrm{TlFeS}_{2}, \mathrm{TIFeSe}_{2}$ в антиферромагнитной фазе. Из рисунка видно, что в обоих кристаллах дно зоны проводимости находится в симметричной точке зоны Бриллюэна Г, а потолок валентной зоны расположен между симметричнами точками $\Gamma$ и $Y$. Этот факт показывает, что края области фундаментального поглощения кристаллов образовываются непрямыми переходами. Ширины запрещенной зоны составляют 0.05 эВ для $\mathrm{TIFeS}_{2}$ и 0.34 эВ для $\mathrm{TIFeSe}_{2}$.

Из рис. 3 видно, что $d$-состояния атомов $\mathrm{Fe}$ спин вверх и спин вниз, компенсируя друг друга, образо- 
вывают антиферромагнитную фазу. Рассчитанные локальные магнитные моменты $\mathrm{Fe}$ в ферромагнитной фазе показывают, что локальный магнитный момент $\mathrm{Fe}$ $\left(\mu=1.21_{\mu \mathrm{Bohr}}\right)$ дает наибольший вклад в полный FMмомент $\left(\mu=1.495_{\mu \text { Bohr }}\right)$ в примитивной ячейке $\mathrm{TlFeS}_{2}$, также расчитанный для примитивной ячейки $\mathrm{TIFeSe}_{2}$ локальный магнитный момент $\mathrm{Fe}\left(\mu=5.642_{\mu \mathrm{Bohr}}\right)$ дает наибольший вклад в полный FM-момент $\left(\mu=5.999_{\mu \text { Bohr }}\right)$.

Локальные магнитные моменты атомов ячеек и суммарный магнитный момент в AFM и FM фазах при разных ориентациях спина приведены в таблице.

Из рассчитанных парциальных плотностей (рис. 3) электронных состояний кристаллов было установлено, что дно зоны проводимости в области $(0.01-2)$ эВ образовано из $3 d$ и $3 p$ электронных состояний атомов $\mathrm{Fe}$ и $\mathrm{S}(\mathrm{Se})$ соответственно. Учитывая тот факт, что в кристалле $\mathrm{TIFeSe}_{2}$ дно зоны проводимости начинается c 0.2 эВ, то $3 p$ состояния атомов $\mathrm{Se}$ будут способствовать формированию диапазона $(0.2-2)$. Также из этих состояний образуются валентные зоны в интервале $(-5--0.02)$ эВ для $\mathrm{TIFeS}_{2}$, а для кристалла $\mathrm{TIFeSe}_{2}$ $3 p$ состояния атомов $\mathrm{Se}$ образуют валентные зоны в интервале $(-5--0.15)$ эВ. Валентная зона в интервале $(-13--14)$ эВ в основном берет свое происхождение из $-3 s$ состояний атомов $\mathrm{S}(\mathrm{Se})$. Частично в образовании этих зон участвуют $\mathrm{Fe}-4 p,-4 s$ состояния атомов.

$5 d$ электронные состояния атомов $\mathrm{Tl}$ делают вклад в образование зон проводимости в интервале (7-9) эВ, валентная зона в области $(-6--7)$ эВ в основном берет свое происхождение из $6 s$ состояний атомов $\mathrm{Tl}$ и частично из $4 s$ состояния атомов $\mathrm{Fe} .6 p$ состояния атомов $\mathrm{Tl}$ делают вклад в образование зоны проводимости в интервале $(3-5)$ эВ. Зона проводимости в интервале $(8-10)$ эВ в основном образуется из $-5 d$ состояния атомов $\mathrm{Tl}$ и частично из $-4 s,-4 p$ состояний атомов $\mathrm{Fe}$.

\section{4. Заключение}

Установлено, что соединения $\mathrm{TlFeS}_{2}, \mathrm{TIFeSe}_{2}$ в антиферромагнитной фазе имеют полупроводниковые свойства с шириной запрещенной зоны $\mathrm{TlFeS}_{2}-0.05$ эВ, $\mathrm{TlFeSe}_{2}-0.34$ эB. Потолок валентной зоны находится между симметричными точками Г и $Y$, дно зоны проводимости находится в симметричной точке зоны Бриллюэна Г. Основной вклад зон в окрестности уровня Ферми дают $-3 d$ состояния атомов $\mathrm{Fe}$ и $-3 p$ состояния атомов $\mathrm{S}(\mathrm{Se})$, частично эти состояния образуются из $-6 s$ состояний атомов $\mathrm{Tl}$.

\section{Список литературы}

[1] A. Kutoglu. Naturwissenchaften B, 61 (3), 125 (1974).

[2] M. Rosenberg, A. Knulle, H. Sabrowsky, C. Platte. Phys. Chem. Sol., 43 (2), 87 (1982).

[3] Р.Г. Велиев, Р.3. Садыхов, Ю.Г. Асадов, Э.М. Керимова, А.И. Джаббаров. Кристаллография, 53 (1), 131 (2008).
[4] Z. Seidov, H.A. Krug von Nidda, J. Hemberger, A. Loidl, G. Sultanov, E. Kerimova, A. Panfilov. Phys. Rev. B, 65, 014433 (2001).

[5] Э.Б. Аскеров, N.T. Dang, А.И. Бескровный, А.И. Мададзада, Д.И. Исмаилов, Р.Н. Мехдиева, S.H. Jabarov, Э.М. Керимова. ФТП, 49 (7), 899 (2015).

[6] Э.Б. Аскеров, А.И. Мададзада, А.И. Бескровный, Д.И. Исмаилов, Р.Н. Мехдиева, С.Г. Джабаров, Э.М. Керимова, Д. Неов. Пов. рент., синх. и нейт. исслед., 12, 5 (2014).

[7] E.B. Asgerov, N.T. Dang, D.I. Ismayilov, S.E. Kichanov, R.N. Mechdiyeva, A.I. Madadzada, S.H. Jabarov, E.M. Kerimov, E.V. Lukin. Mod. Phys. Lett. B, 29, 1350107 (2015).

[8] P. Hohenberg, W. Khon. Phys. Rev. B, 136, B864 (1964).

[9] http:/quantumwise.com/

[10] J. Perdew, K. Burk, Y. Wang. Phys. Rev. B, 54, 16533 (1996).

[11] M. Fuchs, M. Scheffler. Comput. Phys. Commun., 119, 67 (1999).

Редактор Г.А. Оганесян

\section{Calculations electron spectrum and density of states for $\mathrm{TIInS}_{2}$ and $\mathrm{TIFeSe}_{2}$ crystals from first principles}

\author{
N.A. Ismayilova ${ }^{1}$, H.S. Orudjev ${ }^{\mathbf{1}, 2}$, S.H. Jabarov ${ }^{\mathbf{1}, 2}$ \\ ${ }^{1}$ Institute of Physics, \\ Azerbaijan National Academy of Sciences, \\ Az-1143 Baku, Azerbaijan \\ ${ }^{2}$ Azerbaijan Technical University, \\ Az-1073 Baku, Azerbaijan
}

\begin{abstract}
It was established that in the antiferromagnetic phase compounds $\mathrm{TlFeS}_{2}, \mathrm{TIFeSe}_{2}$ have semiconducting properties with a forbidden zone $\mathrm{TlFeS}_{2}-0.05 \mathrm{eV}$ and $\mathrm{TlFeSe}_{2}-0.34 \mathrm{eV}$. The maximum of the valence band is between the symmetrical point $\Gamma$ and $Y$, the bottom of conduction band is in the symmetrical point of the Brillouin zone $\Gamma$. The main contribution of the zones in the vicinity of the Fermi level gives $-3 d$ states of $\mathrm{Fe}$ atoms and $-3 p$ state of $\mathrm{S}(\mathrm{Se})$ atoms, partly these states are formed from $-6 s$ states of $\mathrm{Tl}$ atoms.
\end{abstract}

